OPEN ACCESS

Edited by:

Anil Shanker,

Meharry Medical College,

United States

Reviewed by:

Ranjit Chauhan,

Memorial University of Newfoundland

Canada

Adam J. Gehring,

University Health Network (UHN),

Canada

${ }^{*}$ Correspondence:

Jiming Zhang

jmzhang@fudan.edu.cn

Zhijun Su

su2366@sina.com

tThese authors have contributed equally to this work

Specialty section:

This article was submitted to

T Cell Biology,

a section of the journal

Frontiers in Immunology

Received: 23 October 2018 Accepted: 08 March 2019 Published: 29 March 2019

Citation:

Yu X, Zheng Y, Mao R, Su Z and

Zhang J (2019) BTLA/HVEM

Signaling: Milestones in Research and

Role in Chronic Hepatitis B Virus

Infection. Front. Immunol. 10:617.

doi: 10.3389/fimmu.2019.00617

\section{BTLA/HVEM Signaling: Milestones in Research and Role in Chronic Hepatitis B Virus Infection}

\author{
Xueping $\mathrm{Yu}^{1,2 t}$, Yijuan Zheng ${ }^{1 \dagger}$, Richeng $\mathrm{MaO}^{2}$, Zhijun Su ${ }^{1 *}$ and Jiming Zhang ${ }^{2 *}$ \\ ${ }^{1}$ Department of Infectious Diseases, First Hospital of Quanzhou, Fujian Medical University, Quanzhou, China, ${ }^{2}$ Department of \\ Infectious Diseases, Huashan Hospital, Fudan University, Shanghai, China
}

B- and T-lymphocyte attenuator (BTLA) is an immune-regulatory receptor, similar to CTLA-4 and PD-1, and is mainly expressed on B-, T-, and all mature lymphocyte cells. Herpes virus entry mediator (HVEM)-BTLA plays a critical role in immune tolerance and immune responses which are areas of intense research. However, the mechanisms of the BTLA and the BTLA/HVEM signaling pathway in human diseases remain unclear. This review describes the research milestones of BTLA and HVEM in chronological order and their role in chronic HBV infection.

Keywords: B and T lymphocyte attenuator, herpes virus entry mediator, hepatitis B virus, milestones, T lymphocyte

\section{INTRODUCTION}

Lymphocyte activation is either triggered by the binding of an antigen to its T-cell receptor (TCR) or B-cell receptor (BCR), or by a co-stimulatory or co-inhibitory molecule. T-cells require a co-stimulatory or co-inhibitory molecule for activation, and the quality of T-cell activation is determined by multiple co-signaling molecules. These co-signaling molecules exert both positive stimulatory and negative regulatory functions, and act in a coordinated fashion to maintain homeostasis in the body (1). Co-signaling molecules can be classified into two major families based on their structure. The first is the CD28 immunoglobulin (Ig) superfamily (IgSF), which includes CD28, cytotoxic T-lymphocyte antigen-4 (CTLA-4), inducible costimulatory molecule (ICOS), programmed death-1 (PD-1), and B and T-lymphocyte attenuator (BTLA); and the second is the tumor necrosis factor receptor (TNFR) superfamily (TNFRSF) (2), which includes CD27, CD30, 4-1BB, herpesvirus entry mediator (HVEM), CD40, and OX40 (Table 1). Similar to PD1 and CTLA-4, BTLA inhibits T-cell reactions and cytokine production. Studies on hepatitis B virus (HBV) infection revealed that BTLA is highly expressed in virus-specific T-cells, which have a potent inhibitory effect on events such as T-cell proliferation and cytokine secretion. In this review, we discuss the biological characteristics of BTLA and its ligand and explore their role in chronic HBV infection.

\section{CHRONOLOGICAL MILESTONES IN BTLA RESEARCH}

BTLA, also known as CD272, was first discovered by genetic screening in 2003 for its ability to inhibit Th1 cell expression (3). It is the third new member of the CD28 family discovered after PD1 and CTLA-4. In 2005, HVEM was identified as the specific ligand of BTLA (4). HVEM belongs to the TNFR family and not to the Ig family, thus shattering the perspective that receptors exclusively bind with ligands belonging to the same family. In the same year, the herpes simplex virus type 1 glycoprotein D (HSV1 gD) was found to bind to HVEM from the crystal structure of the BTLA-HVEM complex (5). In the subsequent year, the structure, distribution, biological 
TABLE 1 | Co-stimulatory and inhibitory receptors of the immunoglobulin superfamily and TNFR family.

\begin{tabular}{|c|c|c|c|c|c|}
\hline \multicolumn{3}{|c|}{ Inhibitory molecules } & \multicolumn{3}{|c|}{ Co-stimulatory molecules } \\
\hline Family & Molecule & Ligand & Family & Molecule & Ligand \\
\hline \multirow[t]{11}{*}{$\operatorname{lgSF}$} & PD-1 & PDL1 & $\operatorname{lgSF}$ & CD28 & CD80 \\
\hline & & PDL2 & & & CD86 \\
\hline & CTLA-4 & CD80 & & ICOS & ICOSL \\
\hline & & CD8 & TNFRSF & HVEM & BTLA \\
\hline & BTLA & HVEM & & & LIGHT \\
\hline & Tim3 & Galectin-9 & & 4-1BB & 4-1BBL \\
\hline & TIGIT & PVR, & & OX40 & OX40L \\
\hline & & CD155 & & CD27 & CD70 \\
\hline & & CD112 & & CD30 & CD30L \\
\hline & Lag3 & $\mathrm{MHCll}$ & & CD40 & CD40L \\
\hline & CD160 & HVEM & & GITR & GITRL \\
\hline
\end{tabular}

characteristics, and other aspects of BTLA and HVEM were summarized in a review by Murphy et al. (6), who also found a third motif, Grb-2, in the cytoplasmic domain of BTLA that recruits the PI3K-subunit p85, thus, leading to stimulation of the PI3K signaling pathway and subsequent T-cell activation (7). In 2008, the receptors HSV1 gD, LIGHT (also known as tumor necrosis factor superfamily 14) and CD160, were found to constitute the CD160/BTLA/LIGHT/HVEM signaling regulatory network and to share the same ligand HVEM as BTLA (8). The interactions within the CD160/BTLA/LIGHT/HVEM signaling regulatory network were summarized in the 2009 review by Cai and Freeman (9). Specific BTLA antibody clones such as 6F7 and 6H6 targeting the BTLA-HVEM pathway were summarized in the review by Crawford and Wherry (10). Between 2006 and 2010, the roles and mechanisms of BTLA and its ligands in human diseases [organ transplantation (11), intestinal inflammation (12), rheumatoid arthritis (13), and cancer (14)] and animal models [experimental cerebral malaria (15), mouse pancreatic transplantation (16)] were reported. In 2010, Murphy et al. extensively reviewed the biological characteristics and functional mechanisms of BTLA and its ligands and discussed newer findings (17). A new anticancer therapy based on the blockade of the BTLA signaling pathway was proposed next, which signaled the beginning of a new chapter in cancer intervention (18).

Since HVEM could interact with many co-signaling molecules, Kronenberg et al. proposed that the CD160/BTLA/LIGHT/HVEM signaling regulatory network plays a bidirectional regulatory role in various inflammatory, autoimmune, and infection immune reactions (19). Decreased BTLA levels could induce hyper-activation of T-lymphocytes in HIV patients thereby promoting disease progression (20). In 2012, the HVEM-BTLA signaling pathway was found to be upregulated in the hepatic tissue of HBV-related acute-onchronic liver failure (HBV-ACLF) patients, promoting disease progression (21). In addition, BTLA was also found to promote the development and progression of sepsis through inhibition of the innate immune response (22). In 2013, sirolimus was identified to promote the inhibitory effects of BTLA thereby enabling immune tolerance in kidney allograft (23). BTLA was reported to be a crucial molecular marker in "immunoparalysis" associated with sepsis (24), and it was shown to play a positive regulatory role in viral diseases; for example, mouse hepatitis virus-3 (MHV3) could induce BTLA signaling and cause acute liver failure through phagocyte activation and secretion of the inflammatory molecules TNF- $\alpha$ and FGL2 (25). This study advances our understanding of the conditions that determine the negative or positive regulatory functions of BTLA in humans. In 2015, the BTLA-HVEM signaling pathway was reported to help intestinal parasites (especially Strongyloides stercoralis) maintain an infection (26). In the following year, vaccines blocking the BTLA/CD160 signaling pathway were shown to activate the response of aged $\mathrm{CD}^{+} \mathrm{T}$ cells to the influenza virus (27). Moreover, dendritic cells (DCs) were demonstrated to induce extrathymic T-cell tolerance in peripheral Treg cells through the BTLA-HVEM signaling pathway (28). In 2017, Shen et al. found that the $\mathrm{CD}^{+}{ }^{+} \mathrm{BTLA}^{+}$T-cells isolated lymphocytes from the liver tissue of chronic hepatitis $B$ patients had a negative regulatory effect on Treg cells that helped HBV to avoid immune clearance (29). In 2018, BTLA was elucidated as a marker of a less cytotoxic T-cell subset in diffuse large B-cell lymphoma (30). Our summary listing the research milestones on the BTLA signaling pathway in chronological order aims to distill the information from previous findings and provide more explicit research directions for future studies (Figure 1).

\section{BIOLOGICAL CHARACTERISTICS OF BTLA}

\section{Structure and Distribution of BTLA}

The human BTLA gene, located on chromosome 3 at $3 q 13$, comprises 5 exons with a total length of $870 \mathrm{bp}$ and 3 mRNA splice variants, which encode functional proteins that can be transcribed. Of note, a single nucleotide polymorphism (SNP) of BTLA, rs76844316, was reported to protect against chronic hepatitis B infection (31). BTLA is a type I transmembrane glycoprotein comprising 289 amino acids. Its protein structure is similar to those of CTLA-4 and PD-1 and includes an extracellular domain, transmembrane domain, and cytoplasmic domain (32). The cytoplasmic domain contains three conserved signals: a growth factor receptor-bound protein-2 (Grb-2) recognition motif, an immunoreceptor tyrosine-based inhibitory motif (ITIM), and an immunoreceptor tyrosine-based switch motif (ITSM) (6). ITIM is present in many inhibitory receptors, binding and activating the tyrosine phosphatases SHP-1 and SHP-2, which dephosphorylate tyrosine and inhibit protein tyrosine kinase (PTK)-dependent cell activation (33). The Grb2 recognition motif recognizes the Grb-2 protein, recruits the $\mathrm{PI} 3 \mathrm{~K}$ protein subunit $\mathrm{p} 85$, and stimulates the PI3K signaling pathway, promoting cell proliferation and survival (7). Thus, the BTLA molecule exerts bidirectional regulatory effects: immunosuppressive effects like those on CTLA-4 and PD-1 proteins, and positive stimulatory effects like those on CD28 and ICOS proteins. 


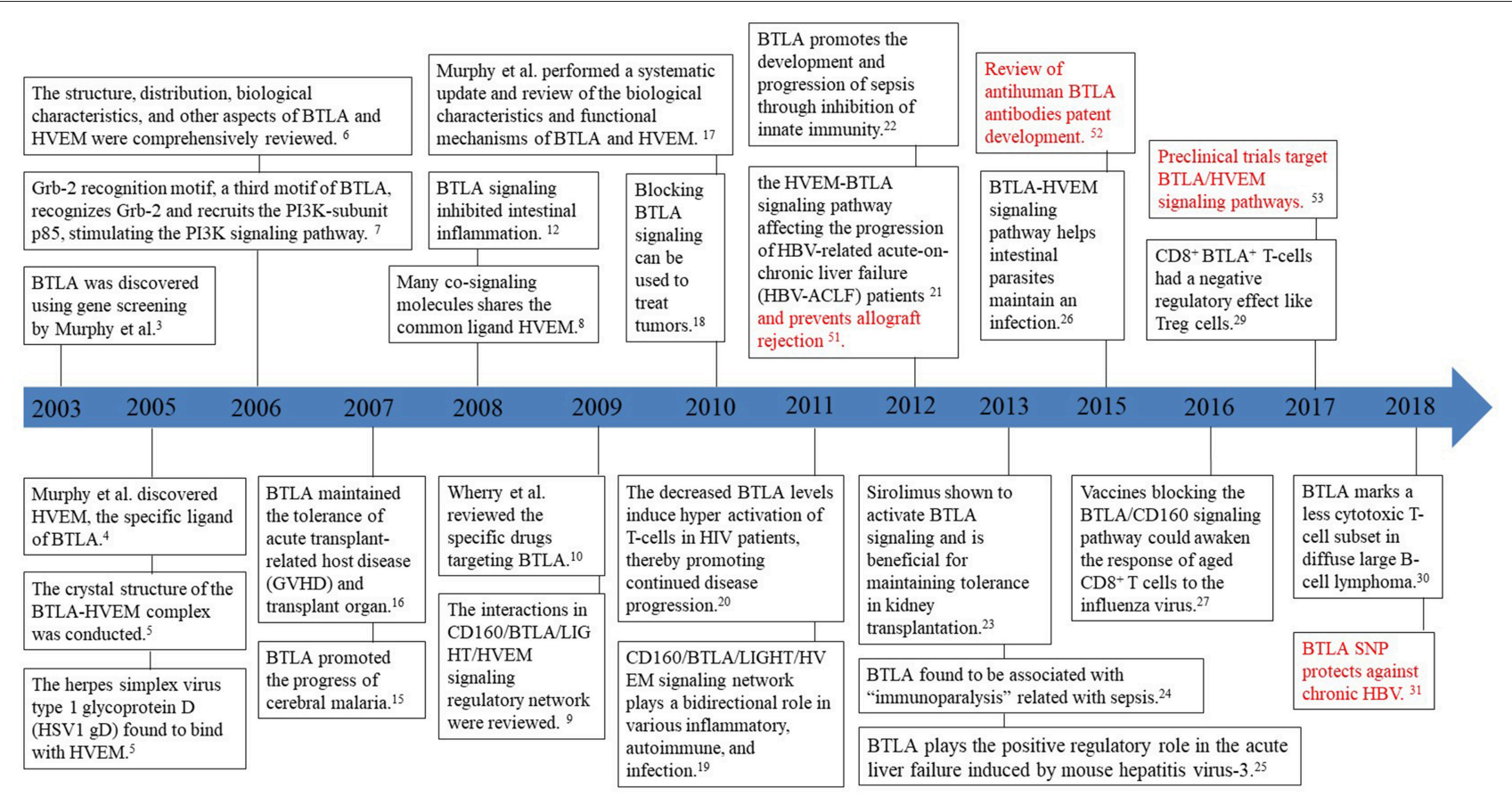

FIGURE 1 | Timeline of milestones in BTLA research.

BTLA is widely expressed in the spleen, thymus, and lymph nodes and has relatively low expression or is even undetectable in the liver, kidney, heart, brain, and other organs. BTLA is constitutively expressed in the CD4/CD8 single-positive Tcells in the mouse thymus (34). Additionally, BTLA is highly expressed in the B-lymphocytes, splenic macrophages, and bone marrow-derived dendritic cells $(35,36)$.

\section{BTLA Ligands}

HVEM, a BTLA-specific receptor, was discovered in 2005 (4). HVEM is expressed in peripheral T- and B-cells, highly expressed in resting $\mathrm{T}$ cells, immature $\mathrm{B}$ cells, and memory $\mathrm{B}$ cells, but downregulated in activated $\mathrm{T}$ and $\mathrm{B}$ cells. Additionally, it is widely expressed in monocytes, dendritic cells, Treg cells, neutrophils, and NK cells $(37,38)$. HVEM binds with many co-signaling molecules, both co-stimulatory and co-inhibitory. The roles that both types of signaling molecules play in signaling pathways also differ and are known as the "molecular switch" models of activation and inhibition. Binding of HVEM to LIGHT or LIGHT- $\alpha$ exerts a positive stimulatory effect, stimulating lymphocyte proliferation, activation, and inducing inflammatory reactions; thus, providing a second stimulatory signal for T cell activation $(4,39)$. Binding of HVEM to BTLA and CD160 exerts an adverse regulatory effect, inhibiting Tand B-lymphocyte activation and proliferation and binding of HVEM to HSV-gD, which can promote HSV infection in target cells (4). Taken together, HVEM provides either an inhibitory or activating signal and bi-directionally regulates host immune function.

\section{FUNCTION OF BTLA IN IMMUNE CELLS}

\section{Function of BTLA in T-lymphocytes}

Resting T-cells express high levels of BTLA and HVEM, and T cell activation increases or decreases BTLA and HVEM expression, respectively. The inhibition of T-cells by BTLA is stronger than the positive stimulatory effect of HVEM on T-cells and prevents the excessive activation of T-cells (40). Importantly, HVEM and BTLA in naive T-cells form a cis-heterodimeric complex, blocking the external CD160 and other co-signaling molecules from binding to HVEM and stimulating the NF- $\mathrm{KB}$ signaling pathway, thereby maintaining T-cell tolerance (41). Other studies have demonstrated that BTLA gene knockdown mice $\left(\mathrm{Btla}^{-/-}\right)$ resist immune tolerance induced by high doses of oral or intravenous ovalbumin (OVA) and show increased infiltration by the inflammatory cells in multiple organs, which induces an autoimmune hepatitis-like disease $(42,43)$. Additionally, Liu et al. could not induce immune tolerance in $\mathrm{Btla}^{-/-}$ mice injected with large doses of OVA, indicating that BTLA plays a vital role in inducing and maintaining $\mathrm{T}$ cell immune tolerance (44).

In addition to inhibiting antigen-specific TCR signalingmediated T-cell proliferation, activation (CD25, CD38), and cytokine (IL-2, IL-4, and IL-10) production (34), the BTLA molecule also crosslinks HVEM on Treg cells to facilitate their immunosuppressant effects (40). Further, BTLA inhibits IgG production by inhibiting secretion of IL- 21 by follicular helper $\mathrm{T}$ cells (Tfh) and plays an essential role in the immunomodulation in body fluids (45). $\gamma \delta \mathrm{T}$ cells play an important role in pathogen clearance and the anticancer process. Interestingly, 
BTLA inhibits $\gamma \delta$ T cell proliferation, and secretion of IL-17, TNF$\alpha$, and other cytokines leading to decreased pathogen clearance and anticancer activity $(46,47)$.

In some instances, binding of BTLA and HVEM mediates immunosuppressive activity and transduces positive signals that promote the survival of effector T cells (33). Tarun et al. used the vaccinia virus to infect mice and found that the BTLAHVEM co-signaling system significantly promotes the survival of antiviral effector $\mathrm{CD}^{+}$T-cells and production of memory cells (48). Competitive stimulation with BTLA antibodies (3C10) can induce IL-10-dependent Treg cell production and helps prolong allogeneic heart transplantation in mice (49). Additionally, BTLA can increase the number and activity of $\gamma \delta \mathrm{T}$ cells and reduce the symptoms of skin inflammation; $\mathrm{Btla}^{-/-}$mice have a reduced number of $\gamma \delta \mathrm{T}$ cells and are susceptible to dermatitis. Moreover, BTLA/HVEM crosslinking was observed to suppress T-cell activation thereby preventing allograft rejection $(21,50)$. Variations of the therapeutic strategy that targets the BTLA-HVEM immune checkpoint pathway using specific antagonist antihuman antibodies has been published in numerous patents, and drugs capable of targeting BTLA-associated signaling pathways such as the HVEM-BTLA-CD160 pathway are currently in preclinical trials $(10,18,51,52)$.

\section{Function of BTLA in B-lymphocytes}

BTLA research has been focused on T-cells, and there are few studies on its function in B-cells. Previous studies have revealed that BTLA is an inhibitory receptor in the BCR signaling pathway. BTLA attenuates the BCR signaling strength by recruiting and phosphorylating the protein tyrosine kinase Syk and downregulating B-cell linker protein, phospholipase $\mathrm{E} 2$, and NF-кB (53). Ware et al. suggested that HVEMBTLA signaling can inhibit CPG-mediated B-cell proliferation and cytokine secretion, and increase stimulatory molecules on their surface; however, this does not affect IL-8 and MIP$1 \beta$ secretion, indicating that BTLA can partially, but not completely, inhibit B cell function (54). However, studies have also shown that BTLA expression in B cells is decreased in elderly patients, leading to reduced responsiveness to the trivalent influenza vaccine, and an inability to produce useful IgG antibodies and mount effective vaccination responses (55). Thus, BTLA can play bidirectional regulatory roles in specific cases.

\section{Function of BTLA in Dendritic Cells}

Latest research demonstrates that HVEM-BTLA signaling plays an important role in maintaining the stability of the internal environment for DCs. Lymphotoxin beta receptor (LT- $\beta$ R) signaling can induce DC proliferation, whereas HVEM-BTLA signaling inhibits their proliferation, indicating that HVEMBTLA signaling can regulate LT- $\beta$ R signaling by feedback and maintain the stability of the internal environment for DCs (56). Interestingly, an adenoviral infection can cause immature DCs to express high levels of CCR7 and exhibit relatively strong migration ability. However, their immune tolerance is relatively poor, and the overexpression of BTLA promotes the maintenance of immune tolerance in these DCs (57). Additionally, BTLA ${ }^{+}$DCs in the thymus increase the expression of CD5 in peripheral T-cells through the BTLA-HVEM signaling pathway and promote the differentiation of these $\mathrm{CD}^{+} \mathrm{T}$ cells into Treg cells; thus, producing extrathymic T-cell tolerance (28).

\section{Function of BTLA in Natural Killer T-cells}

Like B- and T-lymphocytes, BTLA is expressed in the natural killer T (NKT) cells. Nakajima et al. established that BTLA ${ }^{-/-}$ NKT mice secrete more cytokines (IFN- $\gamma$ and IL-4) after $\alpha$ galactosylceramide stimulation and Con A injection compared to wild-type mice, and develop Con A-induced hepatitis more easily(58). However, these phenomena were not observed in $\mathrm{BTLA}^{-/-} \mathrm{NKT}^{-/-}$mice. When BTLA ${ }^{-/-}$NKT and NKT cells were purified in vitro and injected into the $\mathrm{NKT}^{-/-}$mice, mice receiving $\mathrm{BTLA}^{-/-} \mathrm{NKT}$ cells were more susceptible to Con A-induced hepatitis, indicating that BTLA inhibits hepatitis induced by NKT cells. Similarly, Fu et al. also found in a Con Ainduced acute hepatitis model that NKT cells inhibit the release of cytokines (IFN- $\gamma$, IL-2, and IL-4) and liver tissue damage through upregulation of the HVEM-BTLA signaling pathway (59). Additionally, in mouse models of breast cancer, type I NKT cells express high levels of BTLA, and blocking the BTLA signaling pathway may promote infiltration of tumors by NKT cells and inhibit tumor growth (60).

\section{FUNCTION OF BTLA IN CHRONIC HBV INFECTION}

HBV infection severely endangers the health of humans. Globally, there are 240 million patients with HBV infection and every year 0.65 million patients die of HBV-associated endstage liver diseases, whose leading mortality causes include liver cirrhosis (LC), liver failure (LF), primary hepatic cell carcinoma (HCC). China is an endemic zone for HBV infection, and currently, there are 93 million individuals with chronic HBV infection, with $\sim 20$ million chronic hepatitis B (CHB) patients. Thus, HBV infections put a heavy economic burden on the country and its citizens. However, the pathogenic mechanism of chronic HBV infection is not completely understood. Research has demonstrated that the HBVM-BTLA signaling pathway plays an important role in cancer $(14,61)$, intestinal inflammation (12), autoimmune diseases $(4,13)$, viral infection (62), transplant rejection $(11,63)$, and in continuous chronic $\mathrm{HBV}$ infection. In this section, we have provided a current summary of the literature review of BTLA's functions in chronic HBV infection.

\section{Function of BTLA in CHB}

The response of HBV-specific $\mathrm{T}$ cells (CTL) in $\mathrm{CHB}$ patients is extremely weak and can be undetectable. Additionally, the inability to clear HBV leads to continuous infection. Multiple reports suggest that this could be related to an increased expression of T-cell co-inhibitory molecules (e.g., PD-1). However, recent studies demonstrated no significant difference in 
the peripheral blood expression of BTLA in $\mathrm{CD} 4^{+}$and $\mathrm{CD} 8^{+} \mathrm{T}$ cells in CHB patients and healthy individuals, $(64,65)$ and the expression levels were similar in the 4 subtypes of $\mathrm{CD}^{+} \mathrm{T}$ and CD ${ }^{+} \mathrm{T}$ cells (TEM-RA, Tnaïve, Tcm, and Tem) (64). Although these results suggest that BTLA does not contribute to chronic $\mathrm{HBV}$ infection (or CHB immune tolerance); however, there is a difference in BTLA expression levels in the CTL subtypes in the peripheral blood and liver tissue of $\mathrm{CHB}$ patients. In the peripheral blood, BTLA is primarily expressed in the Tcm subtype of T-lymphocytes, whereas BTLA in the liver tissue is primarily expressed in the Tem subtype. This difference may be due to an upregulation of BTLA expression during homing of the peripheral $\mathrm{CD} 8^{+} \mathrm{T}$-cells to the liver that prevents the excessive transition of $\mathrm{CD}^{+} \mathrm{T}$ cells from the $\mathrm{CM}$ stage to the EM stage helping HBV evade immune clearance. Thus, it is believed that $\mathrm{CD}^{+} \mathrm{BTLA}^{+} \mathrm{T}$ cells can negatively regulate Treg cells (29). Critically, during the four different phases of HBV infection [immunotolerant phase, immune clearance phase, nonreactive or minimally (non-) replicative phase, and reactivation phase], the immune reactions produced by HBV are different. Additionally, Zhou et al. established that the frequency of rs76844316 in the G allele of the BTLA gene was decreased in patients with severe $\mathrm{CHB}$, which leads to increased sensitivity to HBV and association with severe disease (31). Therefore, the total BTLA expression level in CHB patients should not be viewed in isolation and BTLA expression in patients at different phases of HBV, across multiple severities of $\mathrm{CHB}$ should be analyzed. However, to our knowledge, no such data have been reported.

\section{Function of BTLA in HBV-LC and HCC}

$\mathrm{CHB}$ is a progressive disease, and the Chinese "Guidelines for the Prevention of Chronic Hepatitis B (2015 edition)" indicates that every year, $\sim 2-10 \%$ of the $\mathrm{CHB}$ patients develop LC and $3-6 \%$ of the LC patients further progress into HCC (66). Liao et al. suggested that BTLA expression levels are significantly upregulated during the progression of CHB from HBV-LC to HCC, but the expression levels of other co-signaling molecules (CD28, ICOS, LIGHT) do not change significantly indicating that BTLA plays an important role in the progression of $\mathrm{CHB}$ (67). BTLA is expressed at high levels in the peripheral blood of patients with $\mathrm{HBV}$-associated HCC and directly correlates with $\mathrm{CD} 4{ }^{+} \mathrm{CD} 25^{+}$Treg cells. These findings indicate that BTLA may have a synergistic effect with $\mathrm{CD} 4^{+} \mathrm{CD} 25^{+}$Treg cells, inhibit Tcell activity and proliferation, and promote the immune evasion of tumors (68). Thus, blocking the BTLA signaling pathway inhibits T-cell function, "awakens" cancer recognition by the immune system, and clears tumor cells. Blockade of the HVEMBTLA signaling pathway has been developed as a new anticancer method $(18,52)$ and has led to more anticancer drugs that target BTLA (Figure 2).

\section{Function of BTLA in HBV-ACLF}

Liver failure is categorized as acute liver failure (ALF), subacute liver failure (SALF), acute-on-chronic liver failure (ACLF), and chronic liver failure (CLF) (69). Clinically, the most common form of $\mathrm{HBV}$-associated liver failure is $\mathrm{HBV}$-associated acute-onchronic liver failure (ACLF) in China, and it has a high mortality

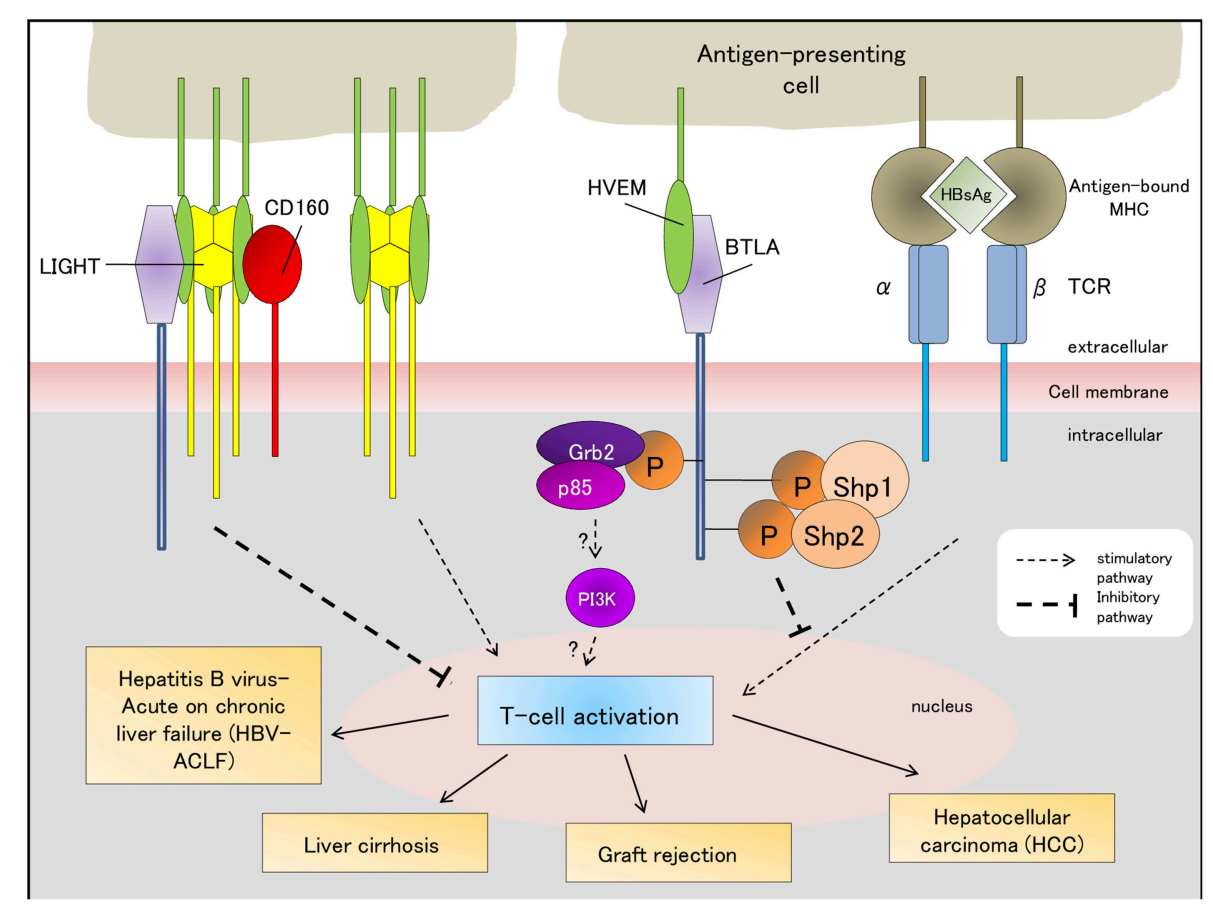

FIGURE 2 | BTLA-associated signaling pathways (stimulatory/inhibitory) regulate the outcomes of HBV-ACLF, liver graft tolerance/rejection, liver cirrhosis and hepatocellular carcinoma. The "?" indicates that the role of the Grb-2 pathway in T-cell-related immune diseases requires further validation and has not been fully characterized yet. HBsAg, Hepatitis B antigen; TCR, T-cell receptor; MCH, major histocompatibility complex. 
rate of $60-80 \%$ (70). ACLF was first coined in 1995 describing a condition arising from two simultaneous insults to the liver, one ongoing and one acute (71). The consensus statements of its definition, diagnosis and management were approved at the 2008 Annual Conference of the APASL in Korea (72), which was defined as an increasingly recognized syndrome characterized by an acute deterioration of liver function and organ/system failure (liver, kidney, brain, coagulation, circulation, and/or respiration) (70).

Since ACLF is a complex and dynamic disease, its diagnostic criteria consists of several components: the cause and timeframe of liver disease development and deterioration following an acute insult, whether patients have pre-existing chronic liver disease, the symptoms of liver failure to determine ACLF severity, and how to assess short- (28-day) and long-term (90-day) prognoses (73). HBV infection is one of the causes of acute liver injury, and BTLA/HVEM signaling contributed to HBV-ACLF pathogenesis (21). The timeframe of acute liver disease development after an acute insult has not been rigorously defined, but several studies have reported it to range from 2 to 8 weeks (73). The role of BTLA/HVEM signaling in determining this timeframe has not been characterized yet. BTLA/HVEM signaling pathway has been shown to prevent T-cell activation thereby promoting malignancy (Figure 2) (68). However, blocking this co-inhibitory pathway limited antitumor response against pre-existing tumor cells (74). The association of BTLA/HVEM signaling with the symptoms of liver failure in ACLF has not been elucidated. Although BTLA/HVEM signaling was shown to be implicated in poor HBV-ACLF outcome (21), further research is necessary to determine its potential as a biomarker for both short- and long-term clinical prognosis.

Currently, there are no effective therapeutic measures, and it is critical to search for early diagnostic markers or targets for pharmacological intervention. Upregulation of PD-1/PD-L1 (75) and BTLA/HVEM (21) pathways in the liver tissue of HBVACLF patients were reported. Additionally, BTLA is primarily expressed in fibrinogen-like protein- 2 and $\mathrm{CD}^{+} 8^{+}$phagocytes and is not expressed in the liver tissue of $\mathrm{CHB}$ patients or healthy individuals. Thus, BTLA has the potential to be a diagnostic

\section{REFERENCES}

1. McGrath MM, Najafian N. The role of coinhibitory signaling pathways in transplantation and tolerance. Front Immunol. (2012) 3:47. doi: 10.3389/fimmu.2012.00047

2. del Rio ML, Kaye J, Rodriguez-Barbosa JI. Detection of protein on BTLA low cells and in vivo antibody-mediated down-modulation of BTLA on lymphoid and myeloid cells of C57BL/6 and BALB/c BTLA allelic variants. Immunobiology. (2010) 215:570-8. doi: 10.1016/j.imbio.2009. 09.008

3. Gavrieli M, Watanabe N, Loftin SK, Murphy TL, Murphy KM. Characterization of phosphotyrosine binding motifs in the cytoplasmic domain of $\mathrm{B}$ and $\mathrm{T}$ lymphocyte attenuator required for association with protein tyrosine phosphatases SHP-1 and SHP-2. Biochem Biophys Res Commun. (2003) 312:1236-43. doi: 10.1016/j.bbrc.2003. 11.070

4. Sedy JR, Gavrieli M, Potter KG, Hurchla MA, Lindsley RC, Hildner K, et al. $\mathrm{B}$ and $\mathrm{T}$ lymphocyte attenuator regulates $\mathrm{T}$ cell activation through marker for HBV-ACLF and provides a theoretical basis for HBVACLF immunotherapy.

\section{CONCLUDING REMARKS}

Since the discovery of BTLA in 2003, multiple studies have established that the HVEM-BTLA signaling pathway plays an essential immunomodulatory role in autoimmune disease, cancer, transplantation, infection, and other diseases. Recent studies on the HVEM-BTLA signaling pathways have unveiled the function and mechanism of BTLA, and targeted anticancer drugs for HVEM-BTLA are emerging. However, whether BTLA participates in inducing functional exhaustion of T-cells and its pathophysiological roles in associated diseases (e.g., HBV-ACLF) remain unknown. It is predicted that studies targeting BTLA will lead to a new revolution in unraveling the immune mechanisms, diagnosis, and treatment of chronic HBV infection.

\section{AUTHOR CONTRIBUTIONS}

$\mathrm{XY}$ and $\mathrm{YZ}$ searched, identified and reviewed the literature, and wrote the manuscript. RM made a table, gave critical comments, and revised the manuscript. JZ and ZS identified and reviewed the literature, wrote the manuscript, and revised the manuscript. All authors have made an intellectual contribution to the manuscript and approved the submission.

\section{FUNDING}

This study was supported by the National Natural Science Foundation of China (81400625, 81670528, and 81672009); Quanzhou high-level talent innovation project (2018C067R); Shanghai Pujiang Program (17PJD005); National Science and Technology Major Project of China (2017ZX10202202 and 2017ZX10202203-007); Natural Science Foundation of Fujian province (2016Y9065), Fujian Provincial Health commission Youth Research Project (2018-1-94, 2018-1-95), and Quanzhou science and technology project (2018Z069, 2018Z074). interaction with herpesvirus entry mediator. Nat Immunol. (2005) 6:90-8. doi: $10.1038 /$ ni1 144

5. Compaan DM, Gonzalez LC, Tom I, Loyet KM, Eaton D, Hymowitz SG. Attenuating lymphocyte activity: the crystal structure of the BTLA-HVEM complex. J Biol Chem. (2005) 280:39553-61. doi: 10.1074/jbc.M507629200

6. Murphy KM, Nelson CA, Sedy JR. Balancing co-stimulation and inhibition with BTLA and HVEM. Nat Rev Immunol. (2006) 6:671-81. doi: 10.1038/nri1917

7. Gavrieli M, Murphy KM. Association of Grb-2 and PI3K p85 with phosphotyrosile peptides derived from BTLA. Biochem Biophys Res Commu. (2006) 345:1440-5. doi: 10.1016/j.bbrc.2006.05.036

8. Kaye J. CD160 and BTLA: LIGHTs out for CD4+ T cells. Nat Immunol. (2008) 9:122-4. doi: 10.1038/ni0208-122

9. Cai G, Freeman GJ. The CD160, BTLA, LIGHT/HVEM pathway: a bidirectional switch regulating T-cell activation. Immunol Rev. (2009) 229:244-58. doi: 10.1111/j.1600-065X.2009.00783.x

10. Crawford A, Wherry EJ. Editorial: therapeutic potential of targeting BTLA. J Leukoc Biol. (2009) 86:5-8. doi: 10.1189/jlb.0209076 
11. Hurchla MA, Sedy JR, Murphy KM. Unexpected role of B and T lymphocyte attenuator in sustaining cell survival during chronic allostimulation. $J$ Immunol. (2007) 178:6073-82. doi: 10.4049/jimmunol.178.10.6073

12. Steinberg MW, Turovskaya O, Shaikh RB, Kim G, McCole DF, Pfeffer K, et al. A crucial role for HVEM and BTLA in preventing intestinal inflammation. J Exp Med. (2008) 205:1463-76. doi: 10.1084/jem.20071160

13. Lin SC, Kuo CC, Chan CH. Association of a BTLA gene polymorphism with the risk of rheumatoid arthritis. J Biomed Sci. (2006) 13:853-60. doi: 10.1007/s11373-006-9113-7

14. Wegiel B, Bjartell A, Culig Z, Persson JL. Interleukin-6 activates PI3K/Akt pathway and regulates cyclin A1 to promote prostate cancer cell survival. Int J Cancer. (2008) 122:1521-9. doi: 10.1002/ijc.23261

15. Lepenies B, Pfeffer K, Hurchla MA, Murphy TL, Murphy KM, Oetzel $\mathrm{J}$, et al. Ligation of $\mathrm{B}$ and $\mathrm{T}$ lymphocyte attenuator prevents the genesis of experimental cerebral malaria. J Immunol. (2007) 179:4093-100. doi: 10.4049/jimmunol.179.6.4093

16. Truong W, Plester JC, Hancock WW, Merani S, Murphy TL, Murphy KM, et al. Combined coinhibitory and costimulatory modulation with anti-BTLA and CTLA4Ig facilitates tolerance in murine islet allografts. Am J Transplant. (2007) 7:2663-74. doi: 10.1111/j.1600-6143.2007.01996.x

17. Murphy TL, Murphy KM. Slow down and survive: enigmatic immunoregulation by BTLA and HVEM. Annu Rev Immunol. (2010) 28:389-411. doi: 10.1146/annurev-immunol-030409-101202

18. Paulos CM, June CH. Putting the brakes on BTLA in T cell-mediated cancer immunotherapy. J Clin Invest. (2010) 120:76-80. doi: 10.1172/JCI41811

19. Shui JW, Steinberg MW, Kronenberg M. Regulation of inflammation, autoimmunity, and infection immunity by HVEM-BTLA signaling. J Leukoc Biol. (2011) 89:517-23. doi: 10.1189/jlb.0910528

20. Zhang Z, Xu X, Lu J, Zhang S, Gu L, Fu J, et al. B and T lymphocyte attenuator down-regulation by HIV-1 depends on type I interferon and contributes to T-cell hyperactivation. J Infect Dis. (2011) 203:1668-78. doi: 10.1093/infdis/jir165

21. Xu H, Cao D, Guo G, Ruan Z, Wu Y, Chen Y. The intrahepatic expression and distribution of BTLA and its ligand HVEM in patients with HBV-related acute-on-chronic liver failure. Diagn Pathol. (2012) 7:142. doi: 10.1186/1746-1596-7-142

22. Shubin NJ, Chung CS, Heffernan DS, Irwin LR, Monaghan SF, Ayala A. BTLA expression contributes to septic morbidity and mortality by inducing innate inflammatory cell dysfunction. J Leukoc Biol. (2012) 92:593-603. doi: 10.1189/jlb.1211641

23. Bai Y, Shi Y, Li Y, Cai B, Zou Y, Wang L. Sirolimus-based regimen promotes inhibitory costimulatory signal of HVEM/BTLA/CD160/LIGHT pathway in allo-renal recipients. Trans Immunol. (2013) 28:38-47. doi: 10.1016/j.trim.2012.11.005

24. Sherwood ER, Hotchkiss RS. BTLA as a biomarker and mediator of sepsisinduced immunosuppression. Crit Care. (2013) 17:1022. doi: 10.1186/cc13143

25. Yang C, Chen Y, Guo G, Li H, Cao D, Xu H, et al. Expression of B and T lymphocyte attenuator (BTLA) in macrophages contributes to the fulminant hepatitis caused by murine hepatitis virus strain-3. Gut. (2013) 62:1204-13. doi: 10.1136/gutjnl-2012-302239

26. Breloer M, Hartmann W, Blankenhaus B, Eschbach ML, Pfeffer K, Jacobs T. Cutting edge: the BTLA-HVEM regulatory pathway interferes with protective immunity to intestinal helminth infection. J Immunol. (2015) 194:1413-6. doi: 10.4049/jimmunol.1402510

27. Dawany N, Parzych EM, Showe LC, Ertl HC. Age-related changes in the gene expression profile of antigen-specific mouse CD8+ T cells can be partially reversed by blockade of the BTLA/CD160 pathways during vaccination. Aging (Albany NY). (2016) 8:3272-97. doi: 10.18632/aging.101105

28. Jones A, Bourque J, Kuehm L, Opejin A, Teague Ryan M, Gross C, et al. Immunomodulatory functions of BTLA and HVEM govern induction of extrathymic regulatory $\mathrm{T}$ cells and tolerance by dendritic cells. Immunity. (2016) 45:1066-77. doi: 10.1016/j.immuni.2016.10.008

29. Wang H, Wu B, Li L, Hu L, Lin J, Jiang C, et al. Hepatic expansion of virus-specific CD8(+)BTLA(+) $\mathrm{T}$ cells with regulatory properties in chronic hepatitis B virus infection. Cell Immunol. (2017) 311:36-45. doi: 10.1016/j.cellimm.2016.10.002

30. Quan L, Lan X, Meng Y, Guo X, Guo Y, Zhao L, et al. BTLA marks a less cytotoxic $\mathrm{T}$-cell subset in diffuse large B-cell lymphoma with high expression of checkpoints. Exp Hematol. (2018) 60:47-56.e1. doi: 10.1016/j.exphem.2018.01.003

31. Tang J, Fei J, Gu C, Liu W, Li M, Zhou C. The influence of B and T lymphocyte attenuator genetic variants on susceptibility to chronic hepatitis B virus infection. Cell Physiol Biochem. (2018) 45:2540-7. doi: 10.1159/000 488272

32. Watanabe N, Gavrieli M, Sedy JR, Yang J, Fallarino F, Loftin SK, et al. BTLA is a lymphocyte inhibitory receptor with similarities to CTLA-4 and PD-1. Nat Immunol. (2003) 4:670-9. doi: 10.1038/ni944

33. Sakoda Y, Park JJ, Zhao Y, Kuramasu A, Geng D, Liu Y, et al. Dichotomous regulation of GVHD through bidirectional functions of the BTLA-HVEM pathway. Blood. (2011) 117:2506-14. doi: 10.1182/blood-2010-08-301325

34. Han P, Goularte OD, Rufner K, Wilkinson B, Kaye J. An inhibitory Ig superfamily protein expressed by lymphocytes and APCs is also an early marker of thymocyte positive selection. J Immunol. (2004) 172:5931-9. doi: 10.4049/jimmunol.172.10.5931

35. Hurchla MA, Sedy JR, Gavrieli M, Drake CG, Murphy TL, Murphy KM. B and $\mathrm{T}$ lymphocyte attenuator exhibits structural and expression polymorphisms and is highly Induced in anergic CD4+ T cells. J Immunol. (2005) 174:337785. doi: 10.4049/jimmunol.174.6.3377

36. Pilat N, Sayegh $\mathrm{MH}$, Wekerle T. Costimulatory pathways in transplantation. Sem Immunol. (2011) 23:293-303. doi: 10.1016/j.smim.2011.04.002

37. Nurieva RI, Chung Y, Hwang D, Yang XO, Kang HS, Ma L, et al. Generation of $\mathrm{T}$ follicular helper cells is mediated by interleukin-21 but independent of T helper 1, 2, or 17 cell lineages. Immunity. (2008) 29:138-49. doi: 10.1016/j.immuni.2008.05.009

38. Steinberg MW, Cheung TC, Ware CF. The signaling networks of the herpesvirus entry mediator (TNFRSF14) in immune regulation. Immunol Rev. (2011) 244:169-87. doi: 10.1111/j.1600-065X.2011.01064.x

39. del Rio ML, Lucas CL, Buhler L, Rayat G, Rodriguez-Barbosa JI. HVEM/LIGHT/BTLA/CD160 cosignaling pathways as targets for immune regulation. J Leukoc Biol. (2010) 87:223-35. doi: 10.1189/jlb.0809590

40. Tao R, Wang L, Murphy KM, Fraser CC, Hancock WW. Regulatory T Cell Expression of herpesvirus entry mediator suppresses the function of $\mathrm{B}$ and T lymphocyte attenuator-positive effector T cells. J Immunol. (2008) 180:6649-55. doi: 10.4049/jimmunol.180.10.6649

41. Cheung TC, Oborne LM, Steinberg MW, Macauley MG, Fukuyama $\mathrm{S}$, Sanjo $\mathrm{H}$, et al. $\mathrm{T}$ cell intrinsic heterodimeric complexes between HVEM and BTLA determine receptivity to the surrounding microenvironment. J Immunol. (2009) 183:7286-96. doi: 10.4049/jimmunol. 0902490

42. Oya Y, Watanabe N, Kobayashi Y, Owada T, Oki M, Ikeda K, et al. Lack of $\mathrm{B}$ and $\mathrm{T}$ lymphocyte attenuator exacerbates autoimmune disorders and induces Fas-independent liver injury in MRL-lpr/lpr mice. Int Immunol. (2011) 23:335-44. doi: 10.1093/intimm/dxr017

43. Oya Y, Watanabe N, Owada T, Oki M, Hirose K, Suto A, et al. Development of autoimmune hepatitis-like disease and production of autoantibodies to nuclear antigens in mice lacking B and T lymphocyte attenuator. Arthritis Rheum. (2008) 58:2498-510. doi: 10.1002/art.23674

44. Liu X, Alexiou M, Martin-Orozco N, Chung Y, Nurieva RI, Ma L, et al. Cutting edge: a critical role of $\mathrm{B}$ and $\mathrm{T}$ lymphocyte attenuator in peripheral T cell tolerance induction. J Immunol. (2009) 182:4516-20. doi: 10.4049/jimmunol.0803161

45. Kashiwakuma D, Suto A, Hiramatsu Y, Ikeda K, Takatori H, Suzuki K, et al. $\mathrm{B}$ and $\mathrm{T}$ lymphocyte attenuator suppresses IL-21 production from follicular Th cells and subsequent humoral immune responses. J Immunol. (2010) 185:2730-6. doi: 10.4049/jimmunol.0903839

46. Bekiaris V, Sedy JR, Macauley MG, Rhode-Kurnow A, Ware CF. The inhibitory receptor BTLA controls gammadelta $\mathrm{T}$ cell homeostasis and inflammatory responses. Immunity. (2013) 39:1082-94. doi: 10.1016/j.immuni.2013.10.017

47. Gertner-Dardenne J, Fauriat C, Olive D. BTLA, a key regulator of Vgamma9Vdelta2 T-cell proliferation. Oncoimmunology. (2013) 2:e25853. doi: 10.4161/onci.25853

48. Flynn R, Hutchinson T, Murphy KM, Ware CF, Croft M, SalekArdakani S. CD8 T cell memory to a viral pathogen requires trans cosignaling between HVEM and BTLA. PLOS ONE. (2013) 8:e77991. doi: 10.1371/journal.pone.0077991 
49. Uchiyama M, Jin X, Matsuda H, Bashuda H, Imazuru T, Shimokawa T, et al. An agonistic anti-BTLA mAb (3C10) induced generation of IL-10-dependent regulatory $\mathrm{CD} 4+\mathrm{T}$ cells and prolongation of murine cardiac allograft. Transplantation. (2014) 97:301-9. doi: 10.1097/01.TP.0000438204.96723.8b

50. Hobo W, Norde WJ, Schaap N, Fredrix H, Maas F, Schellens K, et al. B and $\mathrm{T}$ lymphocyte attenuator mediates inhibition of tumor-reactive CD8+ T cells in patients after allogeneic stem cell transplantation. J Immunol. (2012) 189:39-49. doi: 10.4049/jimmunol.1102807

51. Collin M. Immune checkpoint inhibitors: a patent review (2010-2015). Expert Opin Ther Pat. (2016) 26:555-64. doi: 10.1080/13543776.2016.1176150

52. Torphy RJ, Schulick RD, Zhu Y. Newly emerging immune checkpoints: promises for future cancer therapy. Int J Mol Sci. (2017) 18:E2642. doi: $10.3390 /$ ijms 18122642

53. Vendel AC, Calemine-Fenaux J, Izrael-Tomasevic A, Chauhan V, Arnott D, Eaton DL. B and T lymphocyte attenuator regulates B cell receptor signaling by targeting Syk and BLNK. J Immunol. (2009) 182:1509-17. doi: 10.4049/jimmunol.182.3.1509

54. Thibult ML, Rivals JP, Mamessier E, Gertner-Dardenne J, Pastor S, Speiser $\mathrm{DE}$, et al. CpG-ODN-induced sustained expression of BTLA mediating selective inhibition of human B cells. J Mol Med. (2013) 91:195-205. doi: 10.1007/s00109-012-0943-7

55. Kannan S, Kurupati RK, Doyle SA, Freeman GJ, Schmader KE, Ertl HC. BTLA expression declines on B cells of the aged and is associated with low responsiveness to the trivalent influenza vaccine. Oncotarget. (2015) 6:1944555. doi: 10.18632/oncotarget. 4597

56. De Trez C, Schneider K, Potter K, Droin N, Fulton J, Norris PS, et al. The inhibitory HVEM-BTLA pathway counter regulates lymphotoxin receptor signaling to achieve homeostasis of dendritic cells. J Immunol. (2008) 180:23848. doi: 10.4049/jimmunol.180.1.238

57. Xin H, Zhu J, Miao H, Gong Z, Jiang X, Feng X, et al. Adenovirusmediated CCR7 and BTLA overexpression enhances immune tolerance and migration in immature dendritic cells. BioMed Res Int. (2017) 2017:3519745. doi: $10.1155 / 2017 / 3519745$

58. Iwata A, Watanabe N, Oya Y, Owada T, Ikeda K, Suto A, et al. Protective roles of B and T lymphocyte attenuator in NKT cell-mediated experimental hepatitis. J Immunol. (2010) 184:127-33. doi: 10.4049/jimmunol.0900389

59. Miller ML, Sun Y, Fu YX. Cutting edge: $B$ and $T$ lymphocyte attenuator signaling on NKT cells inhibits cytokine release and tissue injury in early immune responses. J Immunol. (2009) 183:32-6. doi: 10.4049/jimmunol.0900690

60. Sekar D, Govene L, Del Rio ML, Sirait-Fischer E, Fink AF, Brune B, et al. Downregulation of BTLA on NKT cells promotes tumor immune control in a mouse model of mammary carcinoma. Int J Mol Sci. (2018) 19:E752. doi: $10.3390 /$ ijms 19030752

61. Fourcade J, Sun Z, Pagliano O, Guillaume P, Luescher IF, Sander C, et al. $\mathrm{CD} 8(+) \mathrm{T}$ cells specific for tumor antigens can be rendered dysfunctional by the tumor microenvironment through upregulation of the inhibitory receptors BTLA and PD-1. Cancer Res. (2012) 72:887-96. doi: 10.1158/0008-5472.CAN-11-2637

62. Serriari NE, Gondois-Rey F, Guillaume Y, Remmerswaal EB, Pastor S, Messal $\mathrm{N}$, et al. B and T lymphocyte attenuator is highly expressed on CMV-specific $\mathrm{T}$ cells during infection and regulates their function. J Immunol. (2010) 185:3140-8. doi: 10.4049/jimmunol.0902487

63. Tian C, Liu YG, Yan JK, Liu SD, Zhao ST, Wang HW. B- and Tlymphocyte attenuator/herpes virus entry mediator as early indicators for acute rejection following kidney transplantation. Transplant Proc. (2013) 45:157-62. doi: 10.1016/j.transproceed.2012.10.019
64. Cai G, Nie X, Li L, Hu L, Wu B, Lin J, et al. B and T lymphocyte attenuator is highly expressed on intrahepatic $\mathrm{T}$ cells during chronic HBV infection and regulates their function. J Gastroenterol. (2013) 48:1362-72. doi: 10.1007/s00535-013-0762-9

65. Nan XP, Zhang Y, Yu HT, Li Y, Sun RL, Wang JP, et al. Circulating CD4+CD25high regulatory $\mathrm{T}$ cells and expression of $\mathrm{PD}-1$ and BTLA on CD4+ $\mathrm{T}$ cells in patients with chronic hepatitis $\mathrm{B}$ virus infection. Viral Immunol. (2010) 23:63-70. doi: 10.1089/vim. 2009.0061

66. Hou JL, Lai W. The guideline of prevention and treatment for chronic hepatitis B: a 2015 update. Zhonghua Gan Zang Bing Za Zhi. (2015) 23:888-905. doi: 10.3760/cma.j.issn.1007-3418.2015. 12.002

67. Chen J, Wang L, Fu Y, Li Y, Bai Y, Luo L, et al. The co-inhibitory pathway and cellular immune imbalance in the progress of HBV infection. Hepatol Int. (2014) 8:55-63. doi: 10.1007/s12072-013-9464-x

68. Yu HJ, Li J, Shen Q. Expression of BTLA on peripheral lymphocytes in patients with primary carcinoma of the liver. Zhejiang Med. (2012) 34:1563-5.

69. Liver Failure and Artificial Liver Group CSoID, Chinese Medical Association; Severe Liver Disease and Artificial Liver Group, Chinese Society of Hepatology, Chinese Medical Association. Guideline for diagnosis and treatment of liver failure (2018). J Clin Hepatol. (2019) 35:38-44. doi: 10.3969/j.issn.1001-5256.2019.01.007

70. Arroyo V, Moreau R, Jalan R, Gines P, Study E-CCC. acute-on-chronic liver failure: a new syndrome that will re-classify cirrhosis. J Hepatol. (2015) 62(1 Suppl):S131-43. doi: 10.1016/j.jhep.2014.11.045

71. Ohnishi H, Sugihara J, Moriwaki H, Muto Y. [Acute-on-chronic liver failure]. Ryoikibetsu Shokogun Shirizu. (1995):217-9.

72. Sarin SK, Kumar A, Almeida JA, Chawla YK, Fan ST, Garg H, et al. Acuteon-chronic liver failure: consensus recommendations of the Asian Pacific association for the study of the liver (APASL). Hepatol Int. (2009) 3:269-82. doi: 10.1007/s12072-008-9106-x

73. Anand AC, Dhiman RK. Acute on chronic liver failure-what is in a 'definition'? J Clin Exp Hepatol. (2016) 6:233-40. doi: 10.1016/j.jceh.2016.08.011

74. Han L, Wang W, Fang Y, Feng Z, Liao S, Li W, et al. Soluble B and $T$ lymphocyte attenuator possesses antitumor effects and facilitates heat shock protein 70 vaccine-triggered antitumor immunity against a murine TC-1 cervical cancer model in vivo. J Immunol. (2009) 183:7842-50. doi: 10.4049/jimmunol.0804379

75. Cao D, Xu H, Guo G, Ruan Z, Fei L, Xie Z, et al. Intrahepatic expression of programmed death-1 and its ligands in patients with HBVrelated acute-on-chronic liver failure. Inflammation. (2013) 36:110-20. doi: $10.1007 /$ s10753-012-9525-7

Conflict of Interest Statement: The authors declare that the research was conducted in the absence of any commercial or financial relationships that could be construed as a potential conflict of interest.

Copyright (C) 2019 Yu, Zheng, Mao, Su and Zhang. This is an open-access article distributed under the terms of the Creative Commons Attribution License (CC BY). The use, distribution or reproduction in other forums is permitted, provided the original author(s) and the copyright owner(s) are credited and that the original publication in this journal is cited, in accordance with accepted academic practice. No use, distribution or reproduction is permitted which does not comply with these terms. 